\title{
Estudos psicométricos da Escala Eudemon de Bem-Estar Pessoal em adolescentes
}

\author{
Miriam Raquel Wachbolz. Strelhow - Universidade Federal do Rio Grande do Sul, Porto Alegre, Brasil \\ Luciana Rubensan Ourique Masiero - Universidade Federal do Rio Grande do Sul, Porto Alegre, Brasil \\ Bibiana Ramos dos Santos - Universidade Federal do Rio Grande do Sul, Porto Alegre, Brasil \\ Jorge Castellá Sarriera - Universidade Federal do Rio Grande do Sul, Porto Alegre, Brasil \\ Marco Antônio Pereira Teixeira - Universidade Federal do Rio Grande do Sul, Porto Alegre, Brasil
}

\begin{abstract}
Resumo
O presente estudo tem como objetivo avaliar as propriedades psicométricas da escala Eudemon de Bem-Estar Pessoal (EBP) entre adolescentes brasileiros. Participaram do estudo 1.479 adolescentes (64,7\% meninas) entre 12 e 16 anos $(M=14,12$ anos; $D P=1,26)$. Os participantes responderam à EBP e a outros instrumentos de bem-estar. A Análise Fatorial Exploratória $(n=712)$ indicou a presença de dois fatores e a Consistência Interna da Escala mostrou-se adequada $(\alpha=0,767)$. A Análise Fatorial Confirmatória $(n=767)$ demonstrou bons índices de ajuste para a escala com 21 itens. Análises multigrupo indicaram que a estrutura fatorial da escala e as cargas fatoriais dos itens são semelhantes para meninos e meninas. Foram encontradas correlações significativas, com outras escalas de bem-estar, por meio de validação concorrente (correlações de Pearson). Pode-se concluir que o instrumento apresentou evidências de validade, podendo ser utilizado com adolescentes no Brasil. Palavras-chave: escala eudemon de bem-estar pessoal; psicometria; validade estatística; adolescência.
\end{abstract}

\section{Psychometric studies of the Eudemon Scale of Personal Well-being in adolescents}

\begin{abstract}
This study aims to evaluate the psychometric properties of the Eudemon Scale of Personal Well-being (EBP) in Brazilian adolescents. The participants were 1479 adolescents (64.7\% girls) between 12 and 16 years old $(M=14,12, S D=1,26)$. Participants answered the EBP and other instruments of well-being. The Exploratory Factor Analysis $(n=712)$ indicated the presence of two factors and the internal consistency of the scale was adequate $(\alpha=0,767)$. The Confirmatory Factor Analysis $(n=767)$ showed good fit indices of the scale with 21 items. Multigroup analyses indicated that the factor structure of the scale and the factor loadings of the items are similar for boys and girls. Significant correlations of the EBP were found with other scales that assess well-being through concurrent validation (Pearson correlations). It is possible to conclude that the instrument showed evidence of validity and it can be used with Brazilian adolescents.

Keywords: eudemon scale of personal well-being; psychometrics; statistical validity; adolescence.
\end{abstract}

\section{Estudios psicométricos de la Escala Eudemon de Bienestar Personal en adolescentes}

\begin{abstract}
Resumen
El presente estudio tiene como objetivo evaluar las propiedades psicométricas de la escala Eudemon de Bienestar Personal (EBP) entre adolescentes brasileños. Participaron 1.479 adolescentes (64,7\% del sexo femenino) entre 12 y 16 años $(M=14,12$ años; $D T=1,26)$. Los participantes respondieron a la EBP y a otros instrumentos de bienestar. El Análisis Factorial Exploratorio $(n=712)$ indicó la presencia de dos factores, y la consistencia interna de la escala fue adecuada $(\alpha=0,767)$. El Análisis Factorial Confirmatorio $(n=767)$ mostró buenos índices de ajuste para la escala con 21 ítems. Análisis de múltiples grupos señalaron que la estructura factorial de la escala y las cargas factoriales de los ítems son semejantes, tanto para varones como para niñas. Por medio de la validez concurrente (correlación de Pearson) fueron encontradas correlaciones significativas con otras escalas de bienestar. Se concluyó que el instrumento presentó evidencias de validez, y puede ser utilizado con adolescentes en Brasil.

Palabras-clave: escala eudemon de bienestar personal; psicometría; validez estadística; adolescencia.
\end{abstract}

\section{Introdução}

O bem-estar é foco fundamental da Psicologia Positiva, que busca compreender as virtudes e forças pessoais e conhecer os aspectos que podem atuar como fatores protetores e preventivos no desenvolvimento humano (Seligman \& Csikzentmihalyi, 2000). A maioria dos estudos nessa perspectiva tem sido realizada com adultos, e ainda são poucos os que se dedicam aos adolescentes, especialmente no contexto latino-americano (Sarriera et al., 2012).

De uma forma geral, o bem-estar se refere tanto às avaliações subjetivas das pessoas em relação aos diferentes aspectos de suas vidas, quanto às oportunidades estruturais e objetivas, como, por exemplo, a renda. Além disso, deve-se considerar que há variações entre diferentes contextos, bem como em relação à forma de avaliação das pessoas. Por isso, os fatores relacionados 
ao bem-estar devem ser compreendidos de forma específica para cada grupo etário e cultural (Ben-Arieh \& Frones, 2011).

O estudo do bem-estar pode ser considerado a partir de diferentes tradições dentro da Psicologia Positiva, destacando-se as perspectivas hedônica e eudemônica. As duas tradições consideram o bem-estar a partir de avaliações subjetivas, entretanto, enfatizam diferentes aspectos (Keyes, Shmotkin, \& Ryff, 2002). O bem-estar hedônico tem como foco a felicidade, a presença de afetos positivos e a ausência de afetos negativos; enquanto o bem-estar eudemônico está relacionado com a busca de sentido para a vida, o desenvolvimento e a realização pessoal (Deci \& Ryan, 2008).

Em geral, entende-se que a tradição hedônica está relacionada ao estudo do bem-estar subjetivo e a tradição eudemônica, ao estudo do bem-estar psicológico (Casas, 2010). Essa divisão teórica trouxe um debate importante para a Psicologia Positiva, permitindo alguma clareza em um domínio que ainda está em desenvolvimento, com a integração de estudos anteriores e novas descobertas (Fave, Brdar, Freire, Brodrick, \& Wissing, 2011). As pesquisas mais recentes na área têm enfatizado o estudo a partir da tradição hedônica, entretanto, entende-se que o conceito de eudemonia pode auxiliar muito na compreensão do bem-estar (Deci \& Ryan, 2008). Ainda, alguns autores têm apontado para a possibilidade de uma perspectiva multidimensional do bem-estar que inclua aspectos de ambas as concepções (Deci \& Ryan, 2008; Fave et al., 2011).

\section{Instrumentos de avaliação do bem-estar}

Uma das preocupações dos estudiosos do bem-estar é com a disponibilidade de instrumentos de avaliação. Considera-se que as medidas de bem-estar desenvolvidas para uso com crianças e adolescentes são necessárias por considerarem as diferenças entre esses grupos etários e os adultos, incluindo o desenvolvimento intelectual, a maturidade psicossocial, os interesses, a habilidade de leitura e as circunstâncias da vida (Huebner, 2004). Considerando as tradições hedônica e eudemônica, Blasi, Nucera, Cicatiello e Franco (2013) afirmam que do ponto de vista conceitual, a diferença entre as duas perspectivas é bastante clara, porém, é mais difícil identificar os instrumentos específicos, a fim de avaliar essas duas dimensões de uma forma distinta.

Algumas iniciativas nacionais recentes têm sido feitas para disponibilizar instrumentos para a população adolescente, especialmente, em relação ao bem-estar subjetivo. Por exemplo, Bedin e Sarriera (2014) avaliaram as propriedades psicométricas de alguns instrumentos reconhecidos e utilizados internacionalmente, em uma amostra de adolescentes gaúchos e seus pais: Personal Wellbeing Index (PWI, de Cummins, Eckersley, Pallant, van Vugt, \& Misajon, 2003), Brief Multidimensional Student's Life Satisfaction Scale (BMSLSS, de Seligson, Huebner, \& Valois, 2003) e Core Affects Scale (CAS, Russell \& Carroll, 1999). Segabinazi, Giacomoni, Dias, Teixeira e Moraes (2010) desenvolveram uma Escala multidimensional de satisfação de vida, e Segabinazi, Zortea, Zanon, Bandeira, Giacomoni e Hutz (2012) apresentam uma Escala de afetos positivos e negativos para adolescentes brasileiros.

Os instrumentos citados estão relacionados à avaliação do bem-estar subjetivo, partindo de uma perspectiva hedonista. Em termos de avaliação a partir de uma perspectiva eudemônica, não foram encontrados estudos com adolescentes no Brasil que fizessem essa referência. Alguns estudos foram feitos com construtos relacionados a essa perspectiva, como, por exemplo, os trabalhos de Bueno, Strelhow e Câmara (2010) e Aquino, Silva, Figueiredo, Dourado e Farias (2011) que utilizaram o Teste de propósito de vida (PIL-Test) (Harlow, Newcomb, \& Bentler, 1987).

Para a utilização de instrumentos elaborados em outros contextos, além de todo o processo de tradução e adaptação, é necessário também realizar análises estatísticas para confirmar se a estrutura do instrumento é estável quando comparado ao instrumento original, o que envolve a avaliação de sua estrutura fatorial (Borsa, Damásio, \& Bandeira, 2012). Estudos psicométricos permitem avaliar se o instrumento utilizado mede o que se propõe e se está bem estruturado, medindo corretamente. Dessa forma, abrangem tanto a busca por evidências de validade quanto a verificação da fidedignidade do instrumento (Hair, Black, Babin, Anderson, \& Tatham, 2009). Tais análises contribuem para que se obtenha confiança na utilização do instrumento em um determinado contexto. Pretendendo ampliar as possibilidades de investigação sobre o bem-estar na adolescência, o presente estudo tem como objetivo avaliar as propriedades psicométricas da escala Eudemon de Bem-Estar Pessoal entre adolescentes brasileiros.

\section{Escala Eudemon de Bem-Estar Pessoal}

A Escala Eudemon de Bienestar Personal (EBP) foi desenvolvida por Alfredo Fierro e Violeta Cardenal (1996) com a intenção de se obter indicadores confiáveis de saúde mental (comportamental ou psicológica), 
em contraposição ao transtorno psicológico (Fierro \& Rando, 2007). Os autores relacionam o instrumento com a perspectiva eudemônica de bem-estar, por estar relacionado à realização humana, e se propor a medir aspectos para além da perspectiva hedônica (Fierro \& Rando, 2007).

O questionário possuía, inicialmente, 33 itens, com um formato de resposta dicotômica "sim/não". Do total de itens, 14 referiam-se diretamente ao bem-estar, por exemplo: "Passei muito bem as minhas férias" e 19 estavam ligados à ausência de bem-estar, como: "A vida oferece poucas satisfações". Assim, observa-se que um grupo de itens está relacionado a situações mais específicas do cotidiano, e outro à vida da pessoa compreendida de forma global. É importante observar que nenhum dos itens apresenta sentenças com sentido negativo que poderia confundir o respondente. Diversos autores têm mencionado problemas em relação aos itens invertidos ou com negação gramatical, por estes exigirem mais atenção e compreensão por parte do participante (Giacomoni \& Hutz, 2008; Kobarg, Vieira \& Vieira, 2010; Moraes \& Primi, 2002).

Em uma de suas publicações, Fierro (2006) demonstrou que a EBP havia sido utilizada em 14 estudos em seu formato original. A maioria desses estudos foi realizada com adultos, sendo que três deles incluíam amostras de adolescentes (Fierro, 2006). A partir dos resultados obtidos nesses 14 estudos, Fierro pôde concluir que a EBP apresenta uma medida coerente de bem-estar, tendo em vista que apresentou correlações significativas com construtos como autoestima $(r=0,60$, $p<0,01)$, ansiedade $(r=-0,52 \mathrm{a}-0,63, p<0,01)$ e depressão $(r=-0,39$ a $-0,81, p<0,01)$. As correlações obtidas com autoestima e ansiedade podem ser consideradas moderadas, enquanto com depressão variaram de uma correlação fraca à forte, considerando a correlação perfeita igual a um (Hair et al., 2009). Essas correlações então de acordo com resultados que utilizam outras medidas de bem-estar (Fernandes, Vasconcelos-Raposo \& Fernandes, 2012; Garcia, Veiga, Motta, Moura \& Casulari, 2010; Simões, 2013).

Em 2007, Fierro e Rando realizaram um estudo das propriedades psicométricas do instrumento, utilizando uma medida de respostas de quatro pontos ("não, absolutamente", "mais para não", "mais para sim" e "sim, certamente"), diferente do formato original de respostas dicotômicas "sim/não". O estudo realizado com adultos indicou que nove itens apresentavam baixo poder discriminatório. Assim, os autores optaram por eliminá-los e obtiveram uma melhora na consistência interna da escala, chegando a um valor de alfa de Cronbach de 0,92. A confiabilidade para os fatores também foi satisfatória ( $a=0,91$ para "ausência de bem-estar" $\mathrm{e}$ $a=0,79$ para "bem-estar positivo"). Também em uma investigação realizada com crianças e adolescentes, Ferragut e Fierro (2012) encontraram boa consistência interna da escala $(a=0,91)$. No presente estudo, a escala EBP foi traduzida e adaptada em sua versão de 24 itens.

\section{Método}

\section{Participantes}

No total, 1.588 adolescentes entre 12 e 16 anos responderam ao instrumento. Todos eram estudantes de escolas públicas e particulares de Porto Alegre e de quatro cidades do interior do estado do Rio Grande do Sul: Santa Cruz do Sul, Santa Maria, Rio Grande e Passo Fundo. Porém, para esse estudo, considerando-se os pressupostos para as análises realizadas, foram retirados da amostra os participantes que apresentaram mais de $15 \%$ de dados faltantes (três participantes) e aqueles com respostas outliers univariadas (32 participantes) e multivariadas ( 74 participantes).

Assim, a amostra final deste estudo ficou em 1.479 protocolos válidos. A média de idade desses participantes era de 14,12 anos ( $D P=1,26)$, sendo $64,7 \%$ meninas, $53,8 \%$ de escolas públicas e $46,2 \%$ de escolas particulares. Para as análises, os participantes foram divididos aleatoriamente em duas subamostras: grupo 1, com 712 adolescentes, e grupo 2, com 767 adolescentes.

\section{Instrumentos}

Para este estudo foram utilizados, além da Escala Eudemon de Bem-Estar Pessoal, um questionário solicitando informações referentes a dados sociodemográficos, como a idade, o sexo, a escola, a cidade onde mora e outras escalas de bem-estar para o processo de validação da escala Eudemon. As escalas são descritas a seguir:

Escala Eudemon de Bienestar Personal (EBP). Originalmente desenvolvida por Fierro e Cardenal (1996). No presente estudo, foi utilizada a versão de 24 itens da EBP (Fierro \& Rando, 2007), que foi traduzida de sua língua original (espanhol) para o português e, depois, submetida a uma tradução reversa (backtranslation) por um psicólogo fluente em espanhol. As versões foram comparadas e houve concordância entre ambas. Para a aplicação com a amostra brasileira, o instrumento foi adaptado para uma escala de 0 (Não, de nenhuma forma) a 10 (Sim, completamente), pois entende-se 
que, em avaliações relacionadas ao bem-estar, uma escala com mais pontos permite maior sensibilidade do instrumento com uma discriminação mais precisa das respostas dos participantes, principalmente, entre respostas muito altas e intermediárias (Cummins \& Gullone, 2000). No estudo piloto realizado com 30 adolescentes, obteve-se um alfa de Cronbach de 0,76.

Personal Wellbeing Index (PWI). Foi desenvolvido por Cummins et al. (2003) com o objetivo de acessar o aspecto subjetivo da qualidade de vida em grupos variados da população. $\mathrm{O}$ instrumento é composto por sete itens que representam a satisfação com diferentes domínios da vida: saúde, nível de vida, as coisas que tem conseguido, segurança, segurança com o futuro, as relações com outras pessoas e com a comunidade. O índice é respondido em uma escala de 11 pontos, variando de 0 (totalmente insatisfeito) a 10 (totalmente satisfeito). O PWI já foi utilizado em estudos em diferentes países e tem apresentado boa confiabilidade interna, com valores entre 0,70 e 0,85 para o alfa de Cronbach (International Wellbeing Group, 2006). Em estudo com adolescentes brasileiros, a escala obteve um alfa de Cronbach de 0,76 (Bedin \& Sarriera, 2014).

Brief Life Satisfaction Scale (BLSS). Versão breve da Escala de Satisfação com a Vida entre estudantes desenvolvida por Seligson et al. (2003). O instrumento é composto por seis itens, que avaliam a satisfação com a vida em relação aos seguintes aspectos: família, amigos, experiência como estudante, consigo mesmo, com o lugar onde vive e com a vida em geral. O sexto item é considerado como um item único, pois avalia a satisfação da vida globalmente, por isso foram utilizados os cinco primeiros itens para a análise (Bedin \& Sarriera, 2014). A escala é respondida em uma variação de 0 (péssima) a 10 (formidável). Em amostra brasileira, o instrumento com os cinco itens analisados obteve um alfa de Cronbach de 0,72 (Bedin \& Sarriera, 2014).

Item único de Feliciidade Global com a Vida (Happiness Overall Life - HOL), desenvolvido por Fordyce (1988). Os participantes responderam à pergunta "Considerando a sua vida no seu conjunto,você poderia afirmar que se sente", em uma escala de 0 (extremamente infeliz) a 10 (extremamente feliz).

Item único de Satisfação com a Vida (Overall Life Satisfaction - OLS). Os participantes responderam à pergunta: "Atualmente, até que ponto você está satisfeito com toda a sua vida, considerada globalmente?”, em uma escala de 0 (totalmente insatisfeito) a 10 (totalmente satisfeito). O uso de itens únicos na avaliação do bem-estar subjetivo é ressaltado por Campbel, Converse e Rodgers (1976).

\section{Procedimentos}

Os participantes foram contatados em escolas públicas e particulares dos municípios da amostra, sorteadas a partir da lista de escolas disponibilizada pela Secretaria de Educação do Estado do Rio Grande do Sul. As escolas que autorizaram a realização da pesquisa entre seus alunos assinaram um termo de concordância institucional. Após a autorização da escola, os pesquisadores apresentaram a pesquisa aos alunos e entregaram o Termo de Consentimento Livre e Esclarecido (TCLE) para ser preenchido e assinado pelos pais ou responsáveis.

Combinou-se, com a direção das escolas, um horário para entrar nas turmas de sétima e oitava séries do ensino fundamental, e primeira e segunda séries do ensino médio. A aplicação foi de forma coletiva, nas salas de aula, com duração aproximada de 45 minutos, conduzida por dois pesquisadores treinados. Somente participaram aqueles adolescentes que devolveram os TCLEs assinados por eles e seus pais. Essa pesquisa foi aprovada pelo comitê de ética da Universidade Federal do Rio Grande do Sul, e todos os procedimentos atenderam aos critérios éticos, de acordo com a Resolução 196/96 do Conselho Nacional de Saúde do Brasil.

\section{Análise dos dados}

Por meio de análises descritivas dos itens da escala EBP, foram verificados os dados faltantes e respostas outliers. Optou-se pela exclusão dos participantes com mais de $15 \%$ de dados faltantes e os casos com outliers univariados, verificados por meio de escores $z$, considerando-se como valor limite 3,29. Também foram verificados os outliers multivariados, por meio da distância de Mahalanobis. Após esses procedimentos, verificou-se que a amostra final apresentou menos de $2 \%$ de respostas faltantes (missings) e, assim, optou-se pela imputação por regressão desses dados.

A amostra final foi dividida em dois subgrupos para verificar a estrutura fatorial da escala. Inicialmente foram realizadas análises fatoriais exploratórias (AFE) e de confiabilidade $(n=712)$. Em seguida, foram realizadas análises fatoriais confirmatórias (AFC) e AFC multigrupo por sexo para verificar a invariância fatorial $(n=767)$. Como hipótese, esperava-se que as análises realizadas indicassem os dois fatores apontados na literatura - ausência de bem-estar e bem-estar positivo (Fierro \& Rando, 2007). Após esses procedimentos, buscou-se verificar a validade da escala através da validação concorrente, analisando as correlações da escala com outros instrumentos que avaliam bem-estar (PWI, BLSS, OLS e HOL). 


\section{Resultados}

\section{Análise Fatorial Exploratória}

Antes de realizar a Análise Fatorial Exploratória (AFE) foram verificados os pressupostos de homocedasticidade e esfericidade e constatou-se que ambos os critérios foram atendidos $(K M O=0,91$; Teste de Esfericidade de Bartlett $p<0,001)$. A AFE foi realizada com uma amostra de 712 participantes, utilizando-se o procedimento de Análise Paralela, sendo a matriz de dispersão calculada a partir de correlações de Pearson. Optou-se pela rotação oblíqua, tendo em vista a hipótese inicial de existência de correlação entre as dimensões. A análise realizada indicou uma solução de dois fatores, sendo que o primeiro fator apresentou um autovalor de 7,512 e o segundo fator, um autovalor de 2,658. Essa solução corresponde ao proposto pelos autores da escala.

O primeiro fator ficou composto por 15 itens, referindo-se à ausência de bem-estar (a exemplo do item "Se pudesse, mudaria minha vida totalmente"). O segundo foi formado a partir de 09 itens e refere-se à presença do bem-estar, ou bem-estar positivo (a exemplo do item "Sinto-me contente com o que faço a cada dia"). A escala com 24 itens e a solução de duas dimensões são apresentadas na Tabela 1 , com as respectivas cargas fatoriais. Observa-se que foram omitidos os valores abaixo de 0,30 , e que nenhum item obteve carga fatorial acima de $0,30 \mathrm{em}$ mais de um fator.

Nota-se que os itens com as cargas fatoriais mais baixas são os itens 14 (Sinto pena de ter que deixar de ser criança) e 15 (Creio que há pessoas que me querem mal) para o fator 1, e o item 02 (Levanto contente nos dias que não tenho trabalho) para o fator 2. Ainda, identificou-se que a variância explicada por essa solução fatorial foi de $40,74 \%$, tendo sido o primeiro fator responsável por $29,37 \%$, e o segundo por $11,37 \%$.

A confiabilidade da escala foi considerada satisfatória, tendo em vista que o índice alfa de Cronbach para a escala total foi de 0,767 . Da mesma forma, os índices de confiabilidade foram adequados para cada fator, visto que o fator 1 obteve um $\alpha=0,884$ e o fator 2 um $\alpha=0,795$. A análise de confiabilidade indicou ainda um aumento do valor do alfa de Cronbach para o fator 1 caso fosse deletado o item $14(\alpha=0,887)$, e para o fator 2 , caso fosse deletado o item $2(\alpha=0,807)$.

\section{Análise fatorial confirmatória}

Após as análises exploratórias, optou-se pela realização de uma AFC com uma subamostra $(n=767)$, buscando avaliar a adequação da escala por meio dos seguintes indicadores de ajuste: Qui-quadrado, CFI (Índice de Comparação do Ajuste), TLI (Índice de Tucker e Lewis), NFI (Índice de Ajuste Normatizado), RMSEA (Erro Quadrático Médio de Aproximação) e o SRMR (Raiz do Resíduo Médio Padronizado). Consideram-se aceitáveis valores acima de 0,900 para o CFI, TLI e NFI, sendo os melhores os valores próximos a 1, e para o RMSEA e o SRMR, valores abaixo de 0,08 (Hair et al., 2009).

Inicialmente foi testado por meio da AFC o modelo de dois fatores, proposto pelos autores da escala e indicado pela AFE anterior. Para o fator Ausência de bem-estar foram relacionados 15 indicadores, e para o fator Bem-estar positivo, 09 indicadores. Após uma análise inicial desse modelo, optou-se pela retirada de três itens: item 02, 14 e 15, pois eles apresentaram cargas menores que 0,40 , embora significativas. Ao serem retirados esses itens, houve uma melhora no ajuste do modelo. Após a retirada deles, o item 20 foi reavaliado, pois seu peso no fator latente passou de 0,40 para 0,392 . Porém, como sua retirada não apresentou alteração significativa nos índices de ajuste, optou-se por mantê-lo no modelo.

Para o modelo final apresentado na Figura 1, foram realizados acréscimos de covariâncias entre os erros, a partir das indicações dos índices de modificação. Observam-se, na figura, os valores dos parâmetros padronizados para os componentes do modelo (cargas fatoriais, covariâncias entre os erros e covariância entre as variáveis latentes), sendo todos os parâmetros significativos.

A Tabela 2 apresenta os índices de ajuste para os modelos. Observa-se que o modelo inicial composto por todos os itens apresentou índices de resíduos abaixo de 0,08 , valor considerado adequado. Porém, os valores dos índices de ajuste apresentaram-se abaixo de 0,90. Com a retirada dos itens 02,14 e 15 houve uma melhora desses índices de ajuste. Optou-se, ainda, pelo acréscimo de quatro covariâncias entre os erros: dos itens 17 e 18, itens 20 e 21, itens 22 e 24 , e itens 23 e 24 , conforme indicado pelos índices de modificação. Com essas covariâncias, o modelo apresentou melhor ajuste em todos os indicadores analisados.

As cargas fatoriais padronizadas de cada item em seu respectivo fator latente são apresentadas na Tabela 3. Todos os parâmetros apresentados são significativos, variando de 0,392 a 0,792. Para o fator Bem-estar positivo, observa-se que o item 18 "Sinto-me contente com o que faço cada dia" foi o que apresentou a maior carga, 
Tabela 1. Estrutura de Dois Fatores da Escala Eudemon de Bem-Estar Pessoal ( $\mathrm{n}=712)$

\begin{tabular}{|c|c|c|c|}
\hline \multirow{2}{*}{ Itens } & \multicolumn{2}{|c|}{ Cargas fatoriais } & \multirow[t]{2}{*}{$h^{2}$} \\
\hline & Fator 1 & Fator 2 & \\
\hline \multicolumn{4}{|l|}{ Fator 1. Ausência de bem-estar } \\
\hline 23. Ultimamente, muitas coisas tem saído mal para mim. & 0,757 & & 0,620 \\
\hline 21. Se pudesse, mudaria minha vida totalmente. & 0,725 & & 0,532 \\
\hline 09. A vida é muito triste. & 0,711 & & 0,556 \\
\hline 10. Com gosto trocaria de lugar com outra pessoa. & 0,703 & & 0,494 \\
\hline 24. Estou passando por tristezas na vida. & 0,698 & & 0,554 \\
\hline 07. Preferiria fugir de tudo à minha volta. & 0,654 & & 0,485 \\
\hline 04. Sou menos feliz do que esperava quando era mais novo. & 0,633 & & 0,378 \\
\hline 08. Estou tendo má sorte. & 0,627 & & 0,418 \\
\hline 11. Tenho pouca sorte com os/as amigos/as. & 0,598 & & 0,335 \\
\hline 16. Vejo o futuro muito ruim. & 0,588 & & 0,311 \\
\hline 22. Sou daqueles/as que choram de tristeza. & 0,567 & & 0,335 \\
\hline 13. Em casa tenho que passar por situações desagradáveis. & 0,566 & & 0,339 \\
\hline 20. Arrependo-me de muitas coisas que fiz. & 0,550 & & 0,262 \\
\hline 15. Creio que há pessoas que me querem mal. & 0,526 & & 0,258 \\
\hline 14. Sinto pena de ter que deixar de ser criança. & 0,479 & & 0,207 \\
\hline \multicolumn{4}{|l|}{ Fator 2. Bem-estar positivo } \\
\hline 18. Sinto-me contente com o que faço cada dia. & & 0,791 & 0,660 \\
\hline 17. Aproveito muito as pequenas coisas do dia-a-dia. & & 0,685 & 0,414 \\
\hline $\begin{array}{l}\text { 01. Pelas manhãs costumo levantar despertar relaxado/a e com vontade de } \\
\text { começar um novo dia. }\end{array}$ & & 0,648 & 0,388 \\
\hline 03. A vida oferece muitas satisfações. & & 0,641 & 0,498 \\
\hline 19. A vida me traz mais alegrias que tristezas. & & 0,623 & 0,593 \\
\hline 06. Passei muto bem minhas últimas férias. & & 0,585 & 0,345 \\
\hline 05. Adiciono humor à vida. & & 0,582 & 0,335 \\
\hline 12. Agrada-me a casa (ou apartamento) onde vivo. & & 0,567 & 0,327 \\
\hline 02. Levanto contente nos dias que não tenho trabalho. & & 0,386 & 0,133 \\
\hline Confiabilidade ( $\alpha$ de Cronbach) dos fatores & 0,884 & 0,795 & \\
\hline Variância explicada (\%) & 29,37 & 11,37 & \\
\hline
\end{tabular}

enquanto o item 6 "Passei muito bem minhas últimas férias" foi o que apresentou a menor carga. Em relação ao fator Ausência de bem-estar, o item que apresentou maior carga foi o número 9 "A vida é muito triste", e o com a menor carga foi o item 20 "Arrependo-me de muitas coisas que fiz".

\section{Análise fatorial confirmatória multigrupo}

Para verificar a equivalência de parâmetros do modelo proposto da escala entre meninos e meninas, foi conduzida uma AFC multigrupo. Inicialmente, testou-se um primeiro modelo com parâmetros livremente estimados para cada grupo, verificando a adequação do modelo para cada um deles. Em seguida, testou-se um segundo modelo, em que se restringiram as cargas fatoriais para serem iguais aos dois grupos, avaliando a equivalência de significados dos itens para meninos e meninas. Os índices de ajuste comparativos entre os modelos foram analisados, considerando-se a diferença entre o CFI dos modelos. Considera-se que essa diferença $(\Delta C F I)$ não deve ser maior que 0,01 , para que se possa indicar a viabilidade de restringir as cargas 


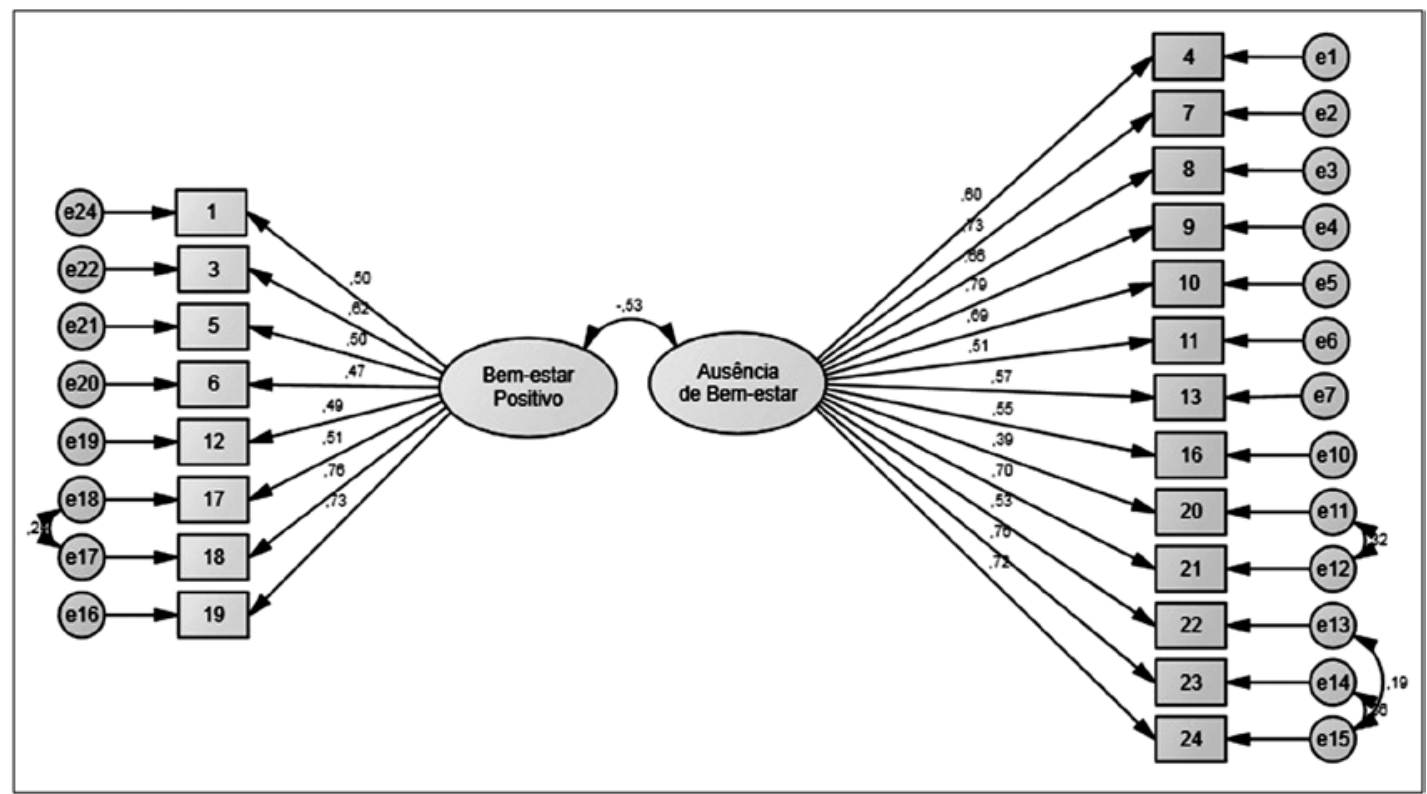

Figura 1. AFC da escala Eudemon de Bem-Estar Pessoal com cargas padronizadas

Tabela 2. Índices de Ajuste para Análise Fatorial Confirmatória da Escala Eudemon de Bem-Estar Pessoal (EBP)

\begin{tabular}{|c|c|c|c|c|c|c|c|c|}
\hline & $\chi^{2}$ & $\mathrm{df}$ & $\mathrm{p}$ & NFI & TLI & CFI & RMSEA (IC) & SRMR \\
\hline EBP 24 itens & 1039,686 & 251 & $<0,001$ & 0,843 & 0,863 & 0,876 & $\begin{array}{c}0,064 \\
(0,060-0,068)\end{array}$ & 0,0557 \\
\hline EBP 21 itens & 822,884 & 188 & $<0,001$ & 0,867 & 0,882 & 0,894 & $\begin{array}{c}0,066 \\
(0,062-0,071)\end{array}$ & 0,0514 \\
\hline $\begin{array}{l}\text { EBP } 21 \text { itens e } 4 \\
\text { covariâncias entre os erros }\end{array}$ & 596,789 & 184 & $<0,001$ & 0,904 & 0,921 & 0,931 & $\begin{array}{c}0,054 \\
(0,049-0,059)\end{array}$ & 0,0463 \\
\hline
\end{tabular}

fatoriais a serem iguais para os diferentes grupos (Milfont \& Fisher, 2010).

As análises indicaram para o modelo 1 (parâmetros livremente estimados) um $X^{2}(368)=793,957$ $(p<0,001), \mathrm{CFI}=0,930$, e RMSEA $=0,039(\mathrm{CI}=0,035$ - 0,043), e para o modelo 2 (cargas fatoriais restringidas) um $X^{2}(387)=859,244(p<0,001), C F I=0,922$, e RMSEA $=0,040(\mathrm{CI}=0,036$ - 0,044). Dessa forma, observa-se que a diferença entre os índices CFI $(\triangle \mathrm{CFI})$ não ultrapassaram $0,01 \mathrm{e}$, por isso, pode-se concluir que tanto a estrutura fatorial da escala como as cargas fatoriais dos itens foram iguais para meninos e meninas.

\section{Correlações da escala EBP com outras escalas de bem-estar}

Após a avaliação da estrutura fatorial da escala e de seu funcionamento com a amostra, buscou-se verificar a sua validade por meio da validação concorrente. Para isso, foram realizadas correlações bivariadas de cada fator da escala EBP, na versão de 21 itens, com dois instrumentos que avaliam bem-estar (PWI e BMLSS) e dois itens únicos, um de satisfação com a vida em geral (OLS) e um de felicidade (HOL).

$\mathrm{Na}$ Tabela 4, observa-se que a correlação entre os dois fatores da escala EBP foi negativa e significativa $(r=-0,401)$. Em relação às correlações entre a escala $\mathrm{EBP}$ e as demais, verifica-se que todas foram significativas, $\operatorname{com} p<0,001$, e na direção esperada: o fator Bem-estar positivo apresentou correlações positivas com os demais instrumentos, enquanto o fator Ausência de bem-estar apresentou correlações negativas. As correlações mais altas foram observadas entre os fatores da escala EBP e a escala BMLSS. 
Tabela 3. Cargas Fatoriais da Escala Eudemon de Bem-Estar Pessoal

\begin{tabular}{lcc}
\hline Item & Fator latente & Cargas fatoriais \\
\hline 1. Pelas manhãs costumo levantar despertar relaxado/a e com vontade de & 0,499 \\
começar um novo dia. & 0,624 \\
3. A vida oferece muitas satisfações. & 0,503 \\
5. Adiciono humor à vida. & Bem-estar & 0,472 \\
6. Passei muto bem minhas últimas férias. & Positivo & 0,491 \\
12. Agrada-me a casa (ou apartamento) onde vivo. & 0,515 \\
17. Aproveito muito as pequenas coisas do dia-a-dia. & 0,759 \\
18. Sinto-me contente com o que faço cada dia. & 0,733 \\
19. A vida me traz mais alegrias que tristezas. & 0,602 \\
\hline 4. Sou menos feliz do que esperava quando era mais novo. & 0,733 \\
7. Preferiria fugir de tudo à minha volta. & 0,657 \\
8. Estou tendo má sorte. & 0,792 \\
9. A vida é muito triste. & 0,692 \\
10. Com gosto trocaria de lugar com outra pessoa. & 0,512 \\
11.Tenho pouca sorte com os/as amigos/as. & Ausência de \\
13. Em casa tenho que passar por situações desagradáveis. & bem-estar & 0,569 \\
16. Vejo o futuro muito ruim. & 0,550 \\
20. Arrependo-me de muitas coisas que fiz. & 0,392 \\
21. Se pudesse, mudaria minha vida totalmente. & 0,705 \\
22. Sou daqueles/as que choram de tristeza. & 0,529 \\
23.Ultimamente, muitas coisas tem saído mal para mim. & 0,755 \\
24. Estou passando por tristezas na vida. & 0,720 \\
\hline
\end{tabular}

Tabela 4. Correlações Entre os Fatores da Escala Eudemon de Bem-Estar Pessoal (EBP) com outros Instrumentos de Bem-Estar

\begin{tabular}{lcccccc}
\hline & $\begin{array}{c}\text { EBP Bem-estar } \\
\text { positivo }\end{array}$ & $\begin{array}{c}\text { EBP Ausência } \\
\text { de bem-estar }\end{array}$ & PWI & BMLSS & OLS & HOL \\
\hline EBP Bem-estar positivo & 1 & $-0,401^{* *}$ & $0,542^{* *}$ & $0,689^{* *}$ & $0,435^{* *}$ & $0,561^{* *}$ \\
EBP Ausência de bem-estar & & 1 & $-0,352^{* *}$ & $-0,469^{* *}$ & $-0,284^{* *}$ & $-0,433^{* *}$ \\
\hline
\end{tabular}

$* * p<0,001$.

\section{Discussão}

Este estudo teve como objetivo analisar as propriedades psicométricas da escala Eudemon de Bem-Estar Pessoal em uma amostra de adolescentes brasileiros, buscando verificar evidências de validade do instrumento. A partir dos resultados apresentados, pode-se afirmar que a escala apresentou evidências de validade e bons indicadores de ajuste para essa amostra.
A AFE realizada com um subgrupo da amostra indicou um agrupamento dos 24 itens originais em dois fatores, conforme proposta dos autores da escala: um fator indicando ausência de bem-estar e o segundo indicando presença de bem-estar positivo. Essa estrutura também foi confirmada pela AFC realizada com o segundo subgrupo da amostra. Conforme indicado pelos autores, essa divisão se deve à formulação dos itens nessas duas direções, positiva e negativa do 
bem-estar. A rotação utilizada comprova a existência de correlação entre os componentes e contribui para sua interpretação (Fierro \& Rando, 2007).

$\mathrm{Na}$ AFE, verificou-se que todos os itens demonstraram contribuir para a escala e apresentaram cargas acima de $0,40 \mathrm{em}$ seu respectivo fator, com exceção do item 02. Esse item, originalmente, refere-se à satisfação da pessoa com os dias em que não tem trabalho. Ao fazer a adaptação para o uso da escala com adolescentes, os autores sugerem que esse item seja modificado, fazendo referência ao estudo, ao invés do trabalho (Fierro, 2006). Porém, para a aplicação na presente pesquisa, optou-se por manter o item original para testá-lo na amostra.

Em relação à confiabilidade do instrumento, os resultados indicaram que está adequada, tanto para a escala total $(\alpha=0,767)$ quanto para os fatores Ausência de bem-estar e Bem-estar positivo ( $a=0,884$ e $a=0,795$, respectivamente). Observa-se que a exclusão dos itens 02 e 14 geraram uma melhora na confiabilidade de seus respectivos fatores.

Com esses dados, pode-se afirmar que a escala apresentou um bom funcionamento para amostra brasileira, mantendo a estrutura fatorial proposta pelos autores, porém com uma ressalva ao item 02. Optou-se, para fins de validação do instrumento, realizar a análise fatorial confirmatória com um segundo subgrupo. Testou-se, assim, o ajuste para esse modelo da escala com a segunda amostra.

Considera-se que a AFC legitimou os resultados obtidos na AFE quanto à estrutura dos 24 itens distribuídos em dois fatores. No entanto, a partir dessa segunda análise, pôde-se confirmar que o item 02 não apresentou um funcionamento adequado e que o instrumento apresentou melhor ajuste, também, com a retirada dos itens 14 e 15 do modelo. A partir dessas reformulações e com o acréscimo das covariâncias de erro indicadas, observou-se que foram encontrados índices adequados de ajuste no modelo confirmatório.

O modelo da escala com 21 itens foi testado novamente em análises multigrupos, que indicaram que a estrutura fatorial da escala, bem como os significados dos itens, foram semelhantes para meninos e meninas. Dessa forma, pode-se afirmar que o modelo proposto para o instrumento é adequado e pode ser utilizado em novos estudos com amostras de ambos os sexos.

Ainda com o objetivo de buscar evidências de validade da escala, optou-se por verificar se seus resultados são compatíveis com outros instrumentos que também se propõem a avaliar o bem-estar (validação concorrente). Assim, optou-se por utilizar, nesse estudo, outros instrumentos de bem-estar adaptados e validados para adolescentes (Bedin \& Sarriera, 2014; Casas et al., 2012).

Especificamente, as escalas PWI e BMLSS foram escolhidas por serem indicadas na literatura como os instrumentos, provavelmente, mais utilizados em estudos com adolescentes (Casas, Tiliouine, \& Figuer, 2013). Um estudo recente realizado com uma amostra de diferentes países utilizando essas duas escalas em conjunto com a escala de Satisfação com a Vida (SWLS, Diener, Emmons, Larsen, \& Griffin, 1985) indicou que, embora essas escalas avaliem domínios diferentes do bem-estar, apresentam altas correlações umas com as outras (Casas et al., 2012). Ressalta-se, ainda, que, embora os quatro instrumentos escolhidos (BMLSS, PWI, HOL e OLS) avaliem o bem-estar de uma perspectiva hedônica, de satisfação com a vida e felicidade, esperava-se que a escala EBP apresentasse correlações significativas com esses instrumentos.

Pode-se observar nos resultados que a validade foi confirmada, visto que as correlações com os demais instrumentos de bem-estar foram significativas e no sentido esperado, considerando-se os fatores Ausência de bem-estar e Bem-estar positivo. Outros estudos de validade com a escala EBP foram realizados, utilizando indicadores clínicos, como depressão, ansiedade, bem como instrumentos de personalidade e inteligência emocional (Fierro, 2006). Nesse sentido, o presente estudo avança ao verificar a relação da escala EBP com outros instrumentos de bem-estar.

\section{Considerações finais}

Nos últimos anos, houve um crescente interesse da avaliação do bem-estar de adolescentes. Para que isso se tornasse possível, houve a necessidade da criação e/ou adaptação de instrumentos específicos para essa população. No Brasil, embora esse seja um tema ainda recente de investigação, alguns instrumentos já foram testados e estão sendo utilizados por diferentes autores (Bedin \& Sarriera, 2014).

Nesse sentido, este estudo buscou averiguar as evidências de validade da escala Eudemon de Bem-Estar Pessoal em uma amostra de adolescentes brasileiros. Uma diferença dessa escala para as demais que têm sido utilizadas refere-se à busca pela avaliação de aspectos eudemônicos do bem-estar (Fierro, 2006), foco apontado como necessário para ampliação das pesquisas de bem-estar (Deci \& Ryan, 2008). Além disso, a proposta 
dos autores é trabalhar em seus itens tanto com episódios bem definidos como, por exemplo, as férias, quanto com aspectos mais amplos da vida, sobre a pessoa e suas relações de uma forma geral (Fierro \& Rando, 2007). Assim, o presente estudo contribui aos estudos do bem-estar, visto que disponibiliza um instrumento com essas características. Destaca-se que, além dos bons resultados psicométricos do instrumento nas análises fatoriais e de confiabilidade, a validade concorrente com outros instrumentos de bem-estar reforça suas evidências de validade.

Entretanto, a ausência de estudos anteriores com instrumentos que avaliem o bem-estar eudemônico, em especial com adolescentes brasileiros, é um fator limitador desse estudo, visto que dificulta sua comparação com outros instrumentos com a mesma fundamentação teórica. Ressalta-se, também, como limitação do estudo, o fato de a escala ter sido aplicada em adolescentes de um único estado brasileiro, considerando a existência de diversos contextos no país e a influência destes no bem-estar (Ben-Arieh \& Frones, 2011). Pesquisas futuras que realizem a aplicação da escala em amostras de adolescentes de diversas regiões do Brasil podem tornar mais evidente seu funcionamento para adolescentes brasileiros.

\section{Referências}

Aquino, T. A. A., Silva, J. P., Figueiredo, A. T. B., Sourado, É. T. S., \& Farias, E. C. S. (2011). Avaliação de uma proposta de prevenção do vazio existencial com adolescentes. Psicologia: Ciência e Profissão, 31(1), 146-159.

Bedin, L. M., \& Sarriera, J. C. (2014). Propriedades psicométricas das escalas de bem-estar: PWI, SWLS, BMSLSS e CAS. Avaliação Psicológica, 13(2), 213-225.

Ben-Arieh, A. \& Frones, I. (2011). Taxonomy for child well-being indicators: a framework for the analysis of the well-being of children. Childhood, 18(4), 460-476.

Blasi, E., Nucera, M., Cicatiello, C., \& Franco, S. (2013). Socio-demographic components of eudaimonic well-being: a survey in an Italian province. Social Indicators Research, 113(1), 451-470.

Borsa, J. C., Damásio, B. F., \& Bandeira, D. R. (2012). Cross-cultural adaptation and validation of psychological instruments: some considerations. Paidéia (Ribeirão Preto), 22(53), 423-432.
Bueno, C. O., Strelhow, M. R. W., \& Câmara, S. G. (2010). Inserção em grupos formais e qualidade de vida entre adolescentes. Psico-USF, 15(3), 311-320.

Campbell, A., Converse, P. E., \& Rodgers, W. L. (1976). The quality of American life: perceptions, evaluations and satisfactions. New York: Russel Sage Foundation.

Casas, F. (2010). El bienestar personal: Su investigación en la infancia y la adolescencia. Encuentros em Psicología Social, 5(1), 85-101.

Casas, F., Sarriera, J. C., Abs, D., Coenders, G., Alfaro, J., Saforcada, E., \& Tonon, G. (2012). subjective indicators of personal Well-Being among adolescents. Performance and results for different scales in latin-language speaking countries: a contribution to the international debate. Child Indicators Research, 5, $1-28$.

Casas, F., Tiliouine, H., \& Figuer, C. (2013). The subjective well-being of adolescents from two different cultures: aplying three versions of PWI in Algeria and Spain. Social Indicators Research, 115(2), 637-651.

Cummins, R., Eckersley, R., Pallant, J., van Vugt, J., \& Misajon, R. A. (2003). Developing a national index of subjective wellbeing: The Australian unity Wellbeing Index. Social Indicators Research, 64(2), 159-190.

Cummins, R. A., \& Gullone, E. (2000). Why we should not use 5-point likert scales: the case for subjective quality of life measurement. Proceedings, Second International Conference on Quality of Life in Cities (pp. 74-93). Singapore: National University of Singapore.

Deci, E. L., \& Ryan, R. M. (2008). Hedonia, eudaimonia and well-being: an introduction. Journal of Hapiness Studies, 9, 1-11.

Diener, E., Emmons, A., Larsen, R. J., \& Griffin, S. (1985). The satisfaction with life scale. Journal of personality assessment, 49(1), 71-75.

Fave, A. D., Brdar, I., Freire, T., Vella-Brodrick, D., \& Wissing, M. P. (2011). The eudaimonic and hedonic components of happiness: Qualitative and quantitative findings. Social Indicators Research, 100, 185-207.

Fernandes, M. G., Vasconcelos-Raposo, J., \& Fernandes, H. M. (2012). Relação entre orientações motivacionais, ansiedade e autoconfiança e bem-estar 
subjetivo em atletas brasileiros. Motricidade, 8(3), 4-18.

Ferragut, M., \& Fierro, A. (2012). Inteligencia emocional, bienestar personal y rendimiento académico en preadolescentes. Revista Latinoamericana de Psicología, 44(3), 95-104.

Fierro, A. (2006). Bienestar personal, adaptación social y factores de personalidad: Estudios com las escalas eudemon. Clínica y Salud, 17(3), 297-318.

Fierro, A., \& Cardenal, V. (1996). Dimensiones de personalidad y satisfacción personal. Revista de Psicología Generaly Aplicada, 49(1), 65-82.

Fierro, A., \& Rando, B. (2007). Escalas Eudemon de Bienestar Personal: características psicométricas. Anuario de Psicologia, 38(3), 401-412.

Fordyce, M. W. (1988). A review of research on the Happiness Measures: A sixty second index of happiness and mental health. Social Indicators Research, 20, 355-381.

Garcia, T. W., Veiga, J. P. R., Motta, L. D. C., Moura, F. J. D., \& Casulari, L. A. (2010). Depressed mood and poor quality of life in male patients with chronic renal failure undergoing hemodialysis. Revista Brasileira de Psiquiatria, 32(4), 369-374.

Giacomoni, C. H. \& Hutz, C. S. (2008). Escala multidimensional de satisfação de vida para crianças: Estudos de construção e validação. Estudos em Psicologia (Campinas), 25 (1), 25-35.

Hair, J. F., Black, W. C., Babin, B. J., Anderson, R. E., \& Tatham, R. L. (2009). Análise multivariada de dados (6 edição). Porto Alegre: Bookman.

Harlow, L. L., Newcomb, M. D., \& Bentler, P. M. (1987). Purpose in life test assessment using latent variable methods. British Journal of Clinical Psychology, 26, 235-236.

Huebner, E. S. (2004). Research on assessment of life satisfaction of children and adolescents. Social Indicators Research, 66, 1-33.

International Wellbeing Group (2006). Personal Wellbeing Index - Adult (PWI-A) Manual (English) $\left(^{\text {th }}\right.$ Edition). Disponível em http://www.deakin.edu. $\mathrm{au} / \mathrm{research} / \mathrm{acqol} /$ instruments/wellbeing-index/ pwi-a-english.pdf

Keyes, C. L., Shmotkin, D., \& Ryff, C. D. (2002). Optimizing well-being: The empirical encounter of two traditions. Journal of Personality and Social Psychology, 82(6), 1007-1022.

Kobarg, A. P. R., Vieira, V., \& Vieira, M. L. (2010). Validação da escala de lembranças sobre práticas parentais. Avaliação Psicológica, 9(1).

Moraes, I. F. \& Primi, R. (2002). Escala de avaliação de tipos psicológicos: Validade e precisão. Psico-USF, $7(1), 25-34$.

Milfont, T. L., \& Fischer, R. (2010). Testing measurement invariance across groups: applications in cross-cultural research. International Journal of Psychological Research, 3(1), 111-121.

Russell, J. A., \& Carroll, J. M. (1999). On the bipolarity of positive and negative affect. Psychological Bulletin, 125(1), 3-30.

Sarriera, J. C., Saforcada, E., Tonon, G., Vega, L. R. L., Mozobancyk, S., \& Bedin, L. (2012). Bienestar subjetivo de los adolescentes: Un estudio comparativo entre Argentina y Brasil. Psychosocial Intervention, 21(3), 273-280.

Segabinazi, J. D., Giacomoni, C. H., Dias, A. C. G, Teixeira, M. A. P., \& Moraes, D. A. O. (2010). Desenvolvimento e validação preliminar de uma escala multidimensional de satisfação de vida para adolescentes. Psicologia: Teoria e Pesquisa, 26(4), 653-659.

Segabinazi, J. D., Zortea, M., Zanon, C., Bandeira, D. R., Giacomoni, C. H., \& Hutz, C. S. (2012). Escala de afeto positivo e negativo para adolescentes: Adaptação, normatização e evidências de validade. Avaliação Psicológica, 11(1), 1-12.

Seligman M. E., \& Csikszentmihalyi, M. (2000). Positive psychology: An introduction. American Psychologist, 55(1), 5-14.

Seligson, J. L., Huebner, E. S., \& Valois, R. F. (2003). Preliminary validation of the Brief Multidimensional Student's Life Satisfaction Scale. Social Indicators Research, 61, 121-145.

Simões, B. M. N. (2013). Ansiedade, satisfação e bem-estar em finalistas e profissionais de psicologia em inicio de carreira (Tese de doutorado não publicada). Universidade dos Açores, Ponta Delgada, Portugal.

Recebido em: 06/03/2014

Primeira Reformulação em: 29/10/2014

Aprovado em: 10/12/2014 
Sobre os autores:

Miriam Raquel Wachholz Strelhow é psicóloga pela Universidade Luterana do Brasil (Canoas, RS), mestre e doutoranda pelo programa de pós-graduação em Psicologia da Universidade Federal do Rio Grande do Sul (UFRGS), e bolsista pelo CNPq, trabalhando principalmente com os seguintes temas: bem-estar de crianças e adolescentes; espiritualidade e coping religioso.

Luciana Rubensan Ourique Masiero é graduada em Psicologia pela Universidade Federal do Rio Grande do Sul (UFRGS), mestre e doutoranda pelo programa de pós-graduação em Psicologia da UFRGS, investigando principalmente as áreas de orientação profissional e desenvolvimento de carreira.

Bibiana Ramos dos Santos é graduada e mestre em Psicologia pela Universidade Federal de Santa Maria (UFSM) e doutoranda pelo programa de pós-praduação em Psicologia da Universidade Federal do Rio Grande do Sul (UFRGS), estudando principalmente psicologia comunitária e bem-estar infantil.

Jorge Castellá Sarriera é doutor em Psicologia Social (Universidade Autonoma de Madrid), com pós-doutorado em Técnicas Estatísticas Multivariadas (Universidade de Barcelona) e em Psicologia Comunitária (University of San Francisco, CA-USA), professor adjunto no Instituto de Psicologia da UFRGS, com interesse em bem-estar na infância e adolescência; inserção no trabalho; desemprego juvenil, transição escola-trabalho, psicologia comunitária e da saúde; imigração, comportamentos de risco; ócio/tempo livre na adolescência; intervenção psicossocial; e programas sociais e avaliação.

Marco Antônio Pereira Teixeira é psicólogo, mestre e doutor em Psicologia pela Universidade Federal do Rio Grande do Sul (UFRGS) e professor no Instituto de Psicologia da UFRGS, com interesse nas áreas de desenvolvimento e aconselhamento de carreira e avaliação psicológica.

Contato com os autores:

Miriam Raquel Wachholz Strelhow

Universidade Federal do Rio Grande do Sul (UFRGS)

Rua Ramiro Barcelos, 2600, sala 122

Porto Alegre, Rio Grande do Sul, Brasil

CEP: 90035-003.

E-mail: raquelwch@gmail.com 